\title{
Efeitos fetais e maternos do propofol, etomidato, tiopental e anestesia epidural, em cesariana eletivas de cadelas
}

\author{
Fetal and maternal effects of propofol, etomidate, tiopental and epidural anesthesia, \\ in bitches elective caesarian section
}

\section{Mário Sérgio Lima de Lavor ${ }^{1}$ Luiz Gonzaga Pompermayer ${ }^{2}$ Shirley Miti Nishiyama ${ }^{1}$ Tatiana Schmitz Duarte ${ }^{1}$ Richard da Rocha Filgueiras ${ }^{1}$ Maria Esther Odenthal ${ }^{1}$}

\section{RESUMO}

$O$ objetivo deste trabalho foi comparar os efeitos entre os fármacos indutores de anestesia como propofol, etomidato e tiopental, e a anestesia epidural com lidocaína seguida de indução, em cadelas submetidas à cesariana, e seus neonatos. Para tanto, foram utilizadas 20 cadelas e 129 filhotes distribuídos em quatro grupos. No grupo 1 (5 cadelas e 39 neonatos), a indução anestésica foi feita com propofol; no grupo 2 (5 cadelas e 25 neonatos), com etomidato; no grupo 3 (5 cadelas e 26 neonatos) com tiopental e no grupo 4, (5 cadelas e 39 neonatos) utilizou-se anestesia epidural e indução com halotano através de máscara. Em todos os casos, a medicação pré-anestésica foi feita com midazolam na dose de $0,22 \mathrm{mg} \mathrm{kg}^{-1}$ via IM, e a manutenção anestésica com halotano em circuito semifechado e concentração inicial de $3 \mathrm{~V} \%$. As variáveis avaliadas nas cadelas foram: temperatura retal, freqüência cardíaca, freqüência respiratória, saturação da oxi-hemoglobina ( $\left.\mathrm{SpO}_{2}\right)$, pressão arterial média. Para a avaliação dos recém-nascidos, foram mensurados: freqüência cardíaca, esforço respiratório, movimentos musculares, coloração das mucosas e irritabilidade reflexa interpretados através do escore de Apgar modificado, bem como a $\mathrm{SpO} \mathrm{O}_{2}$ do neonato. Os resultados mostraram que todos os protocolos foram adequados para as mães com mínimos efeitos sistêmicos. Para o neonato, a utilização de anestesia epidural na mãe, seguida de indução e manutenção com halotano foi superior aos protocolos que usaram agentes injetáveis na indução anestésica.

Palavras-chave: fármacos indutores, depressão fetal, cirurgia.

\begin{abstract}
This work aimed at comparing the anaesthetic inductive effects among drugs such as propofol, etomidate and tiopental as well as the epidural anaesthesia with lidocaine following direct induction in bitches undergone caesarian section and their puppies. For so, 20 bitches and 129 puppies were used and distributed in four groups. group 1 (5 bitches and 39 puppies) the anaesthetic induction was made by using propofol, in group 2 (5 bitches and 25 puppies) by using etomidate, in group 3 ( 5 bitches and 26 puppies) tiopental and in group 4 (5 groups and 39 puppies) epidural anaesthesia and halothane induction through mask. In all cases, the preanaesthetic medication was midazolam in doses of $0.22 \mathrm{mg} \mathrm{kg}^{-1}$ via IM, and the anaesthetic maintance with halothane in semi-closed circuit and initial concentration of $3 \mathrm{~V} \%$. The variables evaluated in the bitches were: rectal temperature, heart rate, breath rate, oxi-hemoglobine saturation $\left(\mathrm{SpO}_{2}\right)$, and mean blood pressure. For the puppies evaluation, it was measured: heart rate, breath effort, muscular movements, mucus color and reflex irritability and also the $\mathrm{SpO}_{2}$. They were interpreted through the modified Apgar score. The results showed that all the protocols were adequate for the mothers with minimum system effects. For the puppies, the epidure anaesthesia usage following the induction and halothane maintance was superior to the protocols that had used injectable agents in the anaesthetic induction.
\end{abstract}

Key words: inductive drugs, foetal depression, surgery.

\footnotetext{
${ }^{1}$ Aluno de Pós-graduação, Departamento de Veterinária, Universidade Federal de Viçosa (UFV), Viçosa, MG. Rua Miguel da Paixão Vasconcelos, 207/202, 29360-000, Castelo, ES, E-mail: mlavor@terra.com.br. Autor para correspondência

${ }^{2}$ Professor, Departamento de Veterinária, UFV.
} 


\section{INTRODUÇÃO}

A cesariana é geralmente um procedimento de emergência e deve contar com técnicas anestésicas seguras para a mãe e os fetos e, ao mesmo tempo, permitir anestesia adequada para a realização da cirurgia em tempo hábil, liberando os filhotes em estado vigoroso (MASTROCINQUE, 2002). Todos os fármacos anestésicos utilizados em cesarianas atravessam rapidamente a barreira placentária, sendo impossível evitar que o feto seja afetado. Ocorre depressão fetal e neonatal de grau variável seguindo a indução anestésica, dependendo do agente indutor especificamente utilizado, de sua dose e do tempo transcorrido desde a indução até a retirada do feto (HELLYER, 1998).

Comparada a outras técnicas de anestesia parenteral ou inalatória, a anestesia regional provavelmente é a que menos produz depressão fetal induzida pelo fármaco (ONG et al., 1989; SHNIDER \& LEVINSON, 1993). As vantagens da anestesia epidural incluem menor exposição do neonato aos fármacos potencialmente depressores e diminuição dos riscos de aspiração pulmonar (SENDAG et al., 1999).

O objetivo deste trabalho foi comparar os efeitos do propofol, etomidato e tiopental, utilizados como fármacos indutores de anestesia, sobre os neonatos e a parturiente e verificar as possíveis vantagens da substituição desses agentes indutores, por anestesia epidural lombossacra, seguida de indução direta com halotano administrado através de máscara, em cesarianas de cadelas.

\section{MATERIAL E MÉTODOS}

Foram utilizadas 20 cadelas adultas gestantes, com idade média de dois anos e meio, proveniente do canil experimental da Universidade Federal de Viçosa, Viçosa - Minas Gerais, pesando $16,77 \pm 8,6 \mathrm{~kg}$ em média, das quais foram obtidos 129 filhotes. As cadelas foram distribuídas aleatoriamente em quatro grupos $(n=5)$, previamente denominados G1, G2, G3 e G4, e receberam os seguintes tratamentos: G1 ( 5 cadelas e 39 filhotes): indução da anestesia com propofol $^{\text {a }}$; $\mathrm{G} 2$ ( 5 cadelas e 25 filhotes): indução com etomidato $^{\text {b; }}$ G3 (5 cadelas e 26 filhotes): indução com tiopental sódico ${ }^{c}$. Todas os fármacos foram administrados por via intravenosa na dose suficiente para permitir a intubação. No G4 (5 cadelas e 39 filhotes), foi feita a administração de lidocaína ${ }^{\mathrm{d}} 2 \%$ na dose de $1 \mathrm{~mL} 4,5 \mathrm{~kg}^{-1}$, por via epidural lombossacra e indução com máscara. Em todos os casos, a medicação pré-anestésica (MPA) foi feita com midazolame na dose de $0,22 \mathrm{mg} \mathrm{kg}^{-1}$ via IM, e a manutenção da anestesia com halotano ${ }^{\mathrm{f}}$, fornecido através de circuito anestésico semi-fechado, diluído em oxigênio e concentração inicial de $3 \mathrm{~V} \%$, medidos através do vaporizador calibrado para halotano. Após instalado o plano anestésico adequado, com bom relaxamento muscular e analgesia, foi iniciada a cirurgia. A remoção do primeiro feto foi feita, como padrão para todos os grupos, 20 minutos após a indução da anestesia.

Foram avaliados os seguintes parâmetros fisiológicos na parturiente: temperatura retal (TR), obtida através de termômetro clínico digital inserido no reto; frequiência respiratória (FR), obtida pela contagem dos movimentos da parede torácica durante um minuto; freqüência cardíaca (FC), estimada por leitura plestimográfica por meio de oxímetro de pulso ${ }^{\mathrm{g}}$, sendo o sensor colocado inicialmente na vulva e após indução do paciente, na língua; saturação da oxihemoglobina $\left(\mathrm{SpO}_{2}\right)$, obtida por meio de oxímetro de pulso; pressão arterial média (PAM), obtida através da mensuração indireta, pelo método oscilométrico, com auxílio de monitor de pressão não invasivo ${ }^{\mathrm{h}}$, sendo o manguito colocado, sobre a artéria mediana, na altura do terço médio do rádio-ulna; período de recuperação, sendo considerado como o tempo compreendido entre o término da administração do agente inalatório e o momento em que o animal assumiu posição quadrupedal; e dose de indução do etomidato, propofol e tiopental. Tais determinações foram feitas nos seguintes momentos: M1 - antes da administração da MPA; M2 - 20 minutos após administração da MPA, imediatamente antes da indução ou administração da anestesia epidural; M3 - logo após a intubação; M4 antes da remoção do primeiro feto; M5 - após retirada do último feto.

Foram utilizadas, para avaliação da vitalidade dos neonatos, a freqüência cardíaca, o esforço respiratório, os movimentos musculares, a resposta aos estímulos na cauda e nas patas e a coloração das mucosas através do escore de Apgar (APGAR, 1953). Este método é amplamente utilizado em medicina humana, sendo neste trabalho, modificado por LAVOR et al. (2002) para que os índices de vitalidade pudessem ser aplicados aos cães recémnascidos (Tabela 1). A interpretação do índice de vitalidade foi obtida pela soma dos escores atribuídos aos sinais clínicos, e assim interpretados: 10 - boas condições clínicas; 7 a 9 - são dispensáveis cuidados especiais; 3 a 6 - requer oxigenação; 0 a 2 - anóxia grave, sendo necessário medidas de ressuscitação. Foi também registrado nos recém-nascidos a $\mathrm{SpO}_{2}$, por meio do oxímetro de pulso, sendo o sensor colocado no membro torácico dos neonatos na região cárpica. 
Tabela 1 - Escore de Apgar modificado.

\begin{tabular}{lccc}
\hline \multicolumn{1}{c}{ Sinais } & 0 & 1 & 2 \\
\hline $\begin{array}{l}\text { Freqüência Cardíaca } \\
\text { Esforço Respiratório }\end{array}$ & Ausente & Menos de 200 & Mais de 200 \\
$\begin{array}{l}\text { Movimentos musculares } \\
\begin{array}{l}\text { Resposta ao estímulo plantar e } \\
\text { caudal por pressão digital }\end{array}\end{array}$ & Ausente & Rento irregular & Movimentação ativa \\
Coloração de mucosas & Nulo & Hipotonia & Movimento e vocalização \\
\hline
\end{tabular}

Fonte: LAVOR et al. (2002).

Os momentos preestabelecidos para avaliação das variáveis fetais foram: MF1 - após dois minutos da retirada do útero (após a desobstrução das vias áreas superiores); MF2 - após cinco minutos da retirada, e MF3 - aos dez minutos de vida extrauterina. Após a retirada dos filhotes, os mesmos foram colocados sobre um colchão térmico-elétrico ${ }^{i}$ que fornecia uma superfície de contato com temperatura máxima de $42^{\circ} \mathrm{C}$.

As variáveis maternas e fetais, bem como o período de recuperação, foram avaliadas estatisticamente pela análise de variância (ANOVA) para experimento inteiramente casualizado, seguida de teste Tukey, executada no programa de Sistema de Análises estatísticas (SAEG). O grau de significância estabelecido foi de $5 \%(\mathrm{p}<0,05)$.

\section{RESULTADOS E DISCUSSÃO}

O uso do midazolam na dose de $0,22 \mathrm{mg} /$ kg está de acordo com diversas citações (THURMON et al., 1996; MUIR III \& HUBBELL, 2001; CORTOPASSI \& FANTONI, 2002) e promoveu um leve efeito sedativo nas parturientes. Em todos os grupos a intubação foi conseguida com facilidade após injeção IV de 4,7 $\pm 1,1 \mathrm{mg}$ $\mathrm{kg}^{-1}$ de propofol, $1,54 \pm 0,5 \mathrm{mg} \mathrm{kg}^{-1}$ de etomidato, ou 9,82 $\pm 2 \mathrm{mg} / \mathrm{kg}$ de tiopental, em médias, assim também como após inalação de halotano a $3 \mathrm{~V} \%$ através da máscara facial por dois minutos, em média. A diminuição da dose comparada com as doses citadas pela literatura pode ser devida às alterações fisiológicas na gestante, em função do aumento da freqüência e do débito cardíaco, tornando maior o fluxo cerebral, propiciando menor tempo de indução anestésica e, conseqüentemente menor dose (MASTROCINQUE, 2002). Outro aspecto importante foi a utilização da MPA. WILDER-SMITH et al. (2001) e MUIR III \& MASON (1989), estudando o propofol e etomidato, respectivamente, verificaram que suas doses são reduzidas com o uso de tranqüilizantes.

A temperatura retal foi reduzida pela anestesia geral, em todos os grupos, porém sem caracterizar hipotermia (menos de $35^{\circ} \mathrm{C}$, segundo WATERMAN, 1975) em nenhum momento (Tabela 2).

Com relação à frequiência respiratória do G3, tratado com tiopental, apresentou um declínio mais acentuado após a intubação (Tabela 2). Provavelmente este efeito esteja relacionado com a ação depressora sobre o centro respiratório bulbar relatado por THURMON et al. (1996) e MUIR III \& HUBBELL (2001), os quais afirmaram que essa ação pode causar hipoventilação e conseqüentemente elevação da $\mathrm{PaCO}_{2}$. A elevada FR observada no G4 em M1 (Tabela 2) pode estar relacionado à excitação inerente ao manuseio pré-operatório. Verifica-se que, após administração da MPA, a FR do G4 diminui consideravelmente, o que pode estar relacionado com a ação depressora do midazolam citada por HOBBS et al. (1996).

As alterações observadas na freqüência respiratória não foram refletidas na saturação de oxihemoglobina que, em todos os grupos, permaneceu durante todo período experimental acima do valor inicial para o paciente anestesiado (Tabela 2). Estes resultados são compatíveis com as afirmações de BOGOD et al. (1988) e PARPAGLIONI et al. (2002), que verificaram que a saturação de oxigênio, tanto materna quanto fetal, aumenta em função da concentração inspirada. Este fato resultou em aumento da $\mathrm{SpO}_{2}$ a partir do início da inalação do oxigênio puro, em todos os grupos.

A frequiência cardíaca, em todos os grupos, manteve-se dentro de limites considerados fisiológicos para a espécie (Tabela 2). Nos momentos seguintes à intubação (M4 e M5), houve declínio não significativo da FC no G1, G2 e G3, o qual pode ser atribuído ao término da excitação inicial e instalação da anestesia cirúrgica (MCKELVEY \& HOLLINGSHEAD, 1994; BALDESSARINI, 1996; MUIR III \& HUBBEL, 2001). A análise em conjunto da freqüência cardíaca e da pressão arterial mostra a interrelação existente entre as variáveis, podendo-se verificar que, em todos os grupos, houve uma relação inversa entre as alterações 


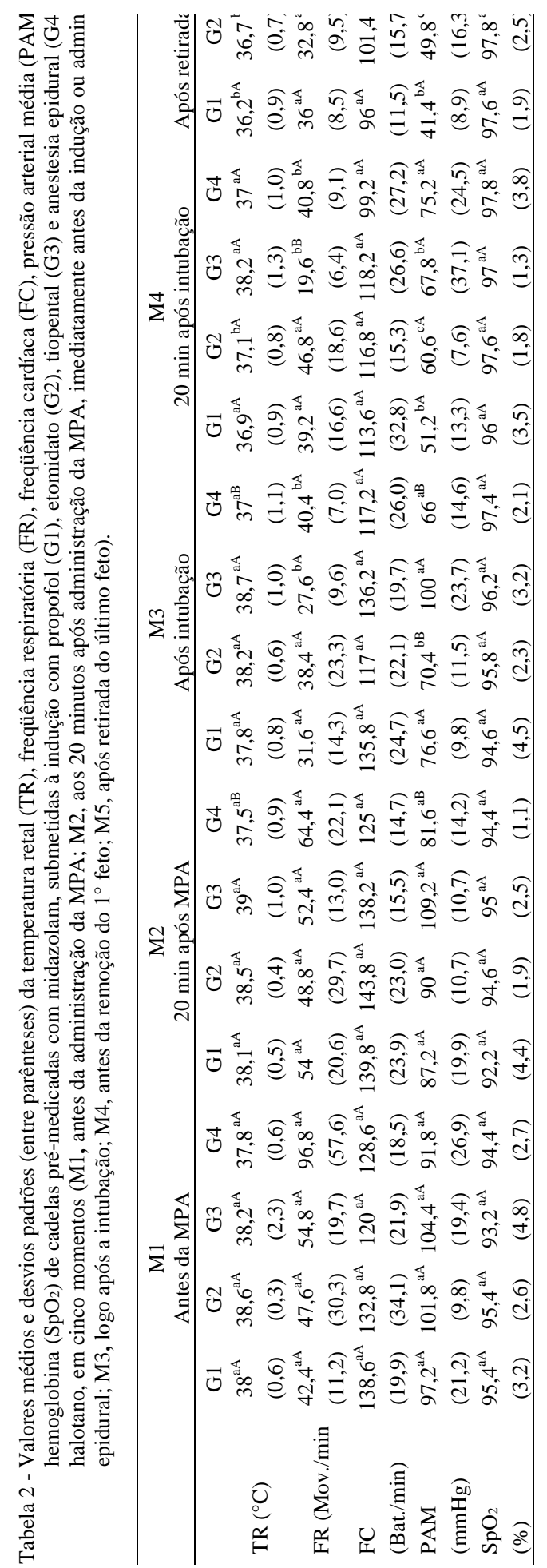

ocorridas na freqüência cardíaca e na pressão arterial, as quais são devidas ao conjunto de efeitos exercidos pelos agentes indutores e o fármaco de manutenção. No grupo onde o fármaco indutor foi substituído pela anestesia epidural (G4) provavelmente a ausência de um agente intravenoso, somada à manutenção de um plano anestésico superficial mantido em $0,5 \mathrm{~V} \%$ de halotano, em decorrência da analgesia promovida pela anestesia epidural, manteve a pressão arterial em nível elevado em M4 e M5, mesmo com uma freqüência cardíaca menor.

Verificou-se bastante semelhança quanto ao período de recuperação encontrado nos grupos 1 (50,6 $\pm 14,1 \mathrm{~min})$ e 2 (40 $\pm 14,4 \mathrm{~min})$. Embora os barbitúricos apresentem recuperação demorada devido ao seu efeito cumulativo, os animais do G3 apresentaram recuperação semelhante a dos grupos G1 e G2 (53,8 $\pm 15,4$ minutos). O maior período de recuperação encontrado no $\mathrm{G} 4(77,4 \pm 15,4 \mathrm{~min})$, foi devido à maior instabilidade dos membros pélvicos em decorrência da permanência de bloqueio nervoso pela anestesia epidural, gerando nesses animais incapacidade para a deambulação.

$\mathrm{Na}$ avaliação dos efeitos da anestesia sobre os fetos, em todos os momentos estudados o G4 apresentou os melhores índices de vitalidade (Figura 1), cuja interpretação dos valores médios, indicou que cuidados especiais eram desnecessários a partir dos cinco minutos após o nascimento. A superioridade deste grupo se deve ao fato de que, além da ausência do fármaco indutor no organismo do neonato, a anestesia epidural promoveu analgesia suficiente para o procedimento cirúrgico na mãe. Isso permitiu que o halotano fosse utilizado, em baixa concentração. Estes resultados coadunam com ONG et al. (1989), SHNIDER \& LEVINSON (1993), DICK (1995) e KRISHNAM et al. (1995) os quais observaram que anestesia regional foi a que produziu menor depressão fetal.

A comparação entre grupos que receberam agentes injetáveis na indução evidencia algumas diferenças. Os neonatos do G1 (propofol) apresentaram o melhor desempenho quando comparados aos dos outros grupos. O índice médio de vitalidade desse grupo indicou que, aos dez minutos, os filhotes não precisavam mais de cuidados especiais. Entretanto, aos dois e aos cinco minutos $76,9 \%$ e $64,1 \%$ respectivamente, dos recémnascidos desse grupo requeriam cuidados especiais. SHORT \& BUFALARI (1999) citaram que o propofol atravessa a barreira placentária com facilidade, mas que as concentrações encontradas no sangue fetal, não têm significado clínico. Citaram ainda que um 


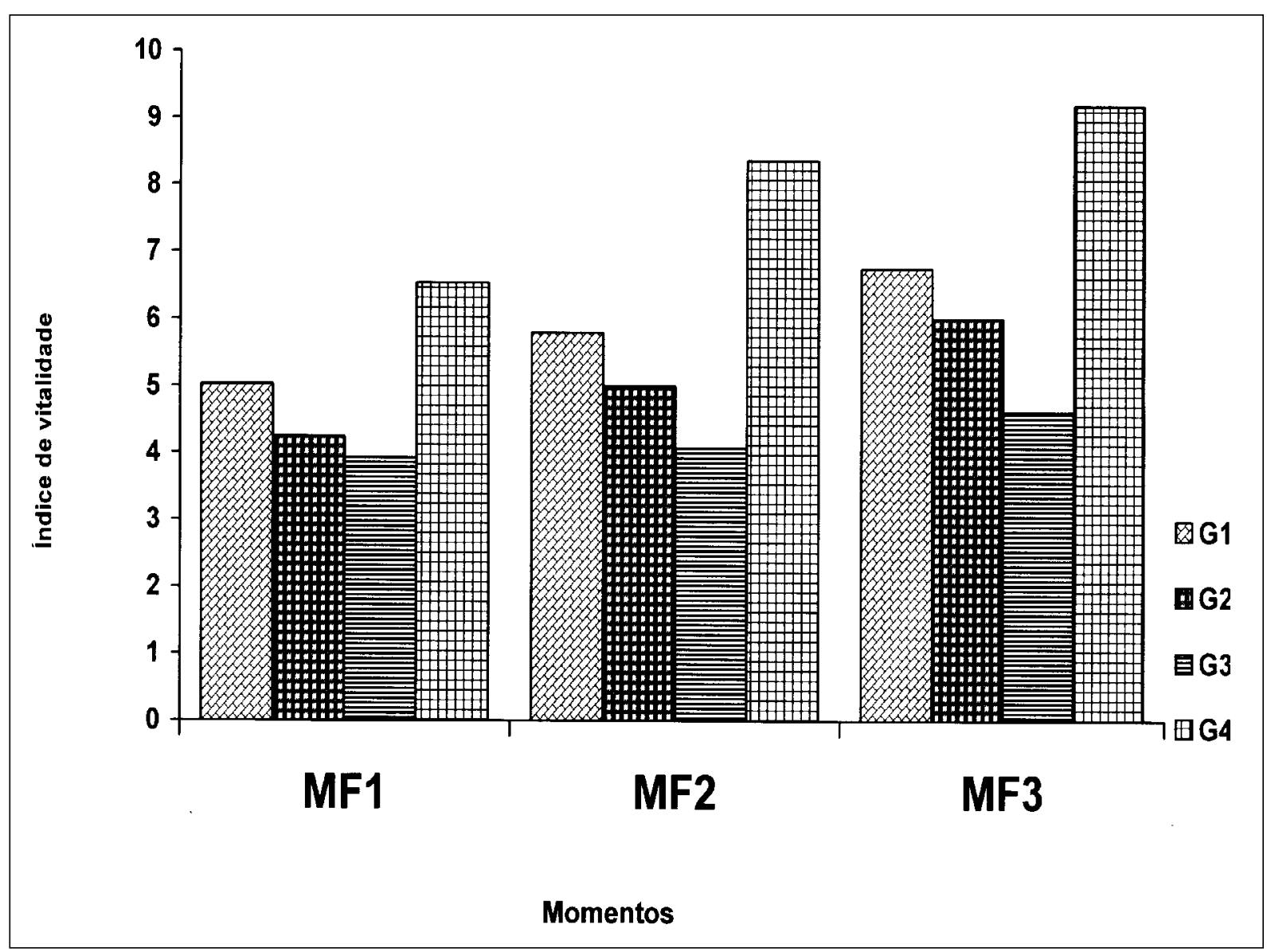

Figura 1- Valores médios do índice de vitalidade obtido através do "escore de Apgar modificado" de cães nascidos de cadelas prémedicadas com midazolam, submetidas à indução com propofol (G1), etomidato (G2), tiopental (G3) e anestesia epidural (G4), e anestesiadas com halotano em três momentos (MF1, após dois minutos da retirada; MF2, após cinco minutos da retirada; MF3, aos dez minutos da retirada)

tempo de 18 a 20 minutos entre a indução e a retirada dos fetos, reduz significativamente a depressão dos neonatos. No presente experimento, houve o cuidado de padronizar em 20 minutos o tempo para a retirada do primeiro feto e a dose de propofol administrada foi abaixo da recomendada, o que leva a crer que, mesmo em condições ideais, de alguma forma, o propofol exerce algum efeito depressivo sobre o feto.

Os neonatos do grupo que recebeu etomidato na indução (G2) apresentaram aos dez minutos, índice de vitalidade intermediário entre o propofol e o tiopental (Figura 1), mas houve necessidade de cuidados especiais por, no mínimo, dez minutos. Entretanto, diferente do que ocorreu com o G3 (tiopental), os neonatos apresentaram alguma melhora dos sinais vitais, no período de dez minutos. Segundo PABLO \& BAILEY (1999), a transferência do etomidato para o feto é incompleta e as concentrações plasmáticas caem rapidamente. Os mesmos autores verificaram uma melhor performance clínica dos neonatos de mães anestesiadas com etomidato do que aqueles nascidos de mães que receberam tiopental. Esta informação é confirmada neste trabalho se for considerado que aos 10 minutos a porcentagem de filhotes com índice menor do que 7 foi de $53,8 \%$ no $\mathrm{G} 2$ e $96,1 \%$ no $\mathrm{G} 3$.

O grupo tratado com tiopental apresentou os menores índices de vitalidade, indicativos de que os neonatos necessitavam de cuidados especiais pelo menos nos 10 primeiros minutos de vida, provavelmente pela depressão prolongada do SNC dos recém-nascidos, que possuem pouca capacidade em metabolizar fármacos (MUIR III \& HUBBELL, 2001). 
Os resultados obtidos através do escore de Apgar modificado mostraram-se confiáveis e foram reforçados pelos valores da $\mathrm{SpO}_{2}$ dos recém-nascidos (Tabela 3), ou seja, os valores mais altos de $\mathrm{SpO}_{2}$ e a melhor evolução clínica durante o período estudado, foram obtidos pelo mesmo grupo de neonatos, cujas mães foram tratadas com anestesia epidural e indução direta. CUNNINGHAM (1992) mostrou que, após as primeiras respirações nos recém-nascidos, a tensão arterial de oxigênio é muito mais alta que no feto. Por não ser encontrado na literatura valor de referência de $\mathrm{SpO}_{2}$ para o neonato canino, optou-se por utilizar esses resultados, principalmente pela possibilidade de poderem servir de subsídios a futuras pesquisas.

Tabela 3 - Valores médios e desvios padrão (entre parênteses) da saturação de oxiemoglobina (\%) de cães recémnascidos de cadelas submetidas à indução com propofol (G1), etomidato (G2), tiopental (G3) e anestesia epidural (G4), e anestesiadas com halotano em três momentos (MF1, após dois minutos da retirada; MF2, após cinco minutos da retirada; $\mathrm{MF3}$, aos dez minutos da retirada)

\begin{tabular}{llll}
\hline & MF1 & MF2 & MF3 \\
\hline G1 & $48,18(24,9)$ & $49,25(26,0)$ & $66,28(27,5)$ \\
G2 & $34,78(23,4)$ & $48,75(22,4)$ & $49,5(19,8)$ \\
G3 & $24,8(26,4)$ & $51,1(19,3)$ & $57,45(22,7)$ \\
G4 & $44,84(23,2)$ & $66,22(22,6)$ & $82,78(16,7)$ \\
\hline
\end{tabular}

\section{CONCLUSÕES}

De acordo com os resultados dessa pesquisa, e nas condições desse experimento, é possível concluir que a utilização de anestesia epidural seguida de indução direta com halotano é superior em promover mínima depressão neonatal e materna aos protocolos anestésicos com propofol, etomidato e tiopental, sendo esse último mais depressor da vitalidade fetal.

\section{FONTES DE AQUISIÇÃO}

aDiprivan - Zeneca Farmacêutica do Brasil - Brasil bEtomidate - Cristália Produtos Químicos e Farmacêuticos Ltda. - Brasil

'Tiopental - Cristália Produtos Químicos e Farmacêuticos Ltda. - Brasil

${ }^{\mathrm{d}}$ Lidocaina 2\% - Hipolabor Farmacêutica Ltda. - Brasil eDormonid - Roche Químicos e Farmacêuticos S. A. - Brasil

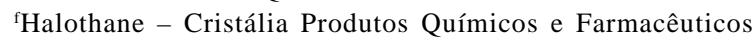
Ltda. - Brasil

${ }^{g}$ Nellcorâ N-100 Pulse Oximeter - USA

${ }^{\text {h} B i o m o n i t o r ~} 4$ - Bio Engenharia de Sistemas e Equip. S. A. Brasil

${ }^{\mathrm{i} C o l c h a ̃ o ~ t e ́ r m i c o ~-~ T e r w a y ~}{ }^{\circledR}$ - Calor e conforto.

\section{REFERÊNCIAS BIBLIOGRÁFICAS}

APGAR, V. A proposal for a new method of evaluation of the newborn infant. Curr Res Anesth Analg v.32, n.4, p.260267,1953

BALDESSARINI, R.J. Fármacos e tratamento dos distúrbios psiquiátricos: psicose e ansiedade. In: HARDMAN, J.G.; LIMBIRD, L.E. Goodman \& Gilman - as bases farmacológicas da terapêutica. Rio de janeiro : Guanabara Koogan, 1996. p.290-313.

BOGOD, D.G. et al. Maximum $\mathrm{F}_{\mathrm{IO} 2}$ during caesarean section. Br J Anaesth, v.61, n.3, p.255-262, 1988.

CORTOPASSI, S.R.G.; FANTONI, D.T. Medicação préanestésica. In: ____ Anestesia em cães e gatos. São Paulo : Rocca, 2002. Cap.13, p.151-158.

CUNNINGHAM, J.G. Transporte fetal e neonatal de oxigênio. In: ______. Tratado de fisiologia veterinária. Rio de Janeiro : Guanabara Koogan, 1992. Cap.49, p.413-417.

DICK, W.F. Anaesthesia for caesarean section (epidural and general): effects on the neonate. Eur Journal of Obstetrics \& Gynecology and Reproductive Biology, Suppl.59, p.s61-s67, 1995.

HELLYER, P.W. Anestesia para operação cesariana. In: SLATTER, D. Manual de cirurgia de pequenos animais. São Paulo : Manole, 1998. V.2, p.2714-2718.

HOBBS, W.R. et al. Hipnóticos e sedativos; etanol. In: HARDMAN, J.G.; LIMBIRD, L.E. Goodman \& Gilman as bases farmacológicas da terapêutica. Rio de janeiro : Guanabara Koogan, 1996. p.263-289.

KRISHNAM, L. et al. Neonatal effects of anesthesia for caesarean section. Indian J Pediatr, v.62, n.1, p.109-113, 1995.

LAVOR, M.S.L. et al. Efeitos do propofol, etomidato, tiopental e anestesia epidural sobre os neonatos de cesarianas eletivas de cadelas. Revista Bras Ci Vet, sup1. v.9, n.1, p.349-351, 2002

MASTROCINQUE, S. Anestesia em ginecologia e obstetrícia. In: FANTONI, D.T.; CORTOPASSI, S.R.G. Anestesia em cães e gatos. São Paulo : Rocca, 2002. Cap.23, p.231-238.

MCKElVEY, D.; HOLlingSEAD, K.W. Small animal anesthesia - Canine and feline pratice. Missouri : Mosby - Year Book, 1994. 332p.

MUIR III, W.W.; HUBBELL, J.A. Anestesia para cesarianas. In: _. Manual de anestesia veterinária. 3.ed. Porto Alegre: Artmed, 2001. Cap.23, p.290-296.

MUIR III, W.; MASON, D. Side effects of etomidate in dogs. J Am Vet Med Assc, v.194, n.10, p.1430-1434, 1989.

ONG, B.Y. et al. Anesthesia for cesarean section - effects on neonates. Anesth Analg, v.68, p.270-275, 1989.

PABLO, L.S.; BAILEY, J.E. Etomidate and telazol. Vet Clin Nort Amer: Small Anim Pratice, v.29, n.3, p.779792, 1999. 
PARPAGLIONI, R. et al. Intraoperative fetal oxygen saturation during caesarean section: general anaesthesia using sevoflurane with either $100 \%$ oxygen or $50 \%$ nitrous oxide in oxygen. Eur J Anaesth v.19, p.115-118, 2002.

SENDAG, F. et al. Comparison of epidural and general anaesthesia for elective cesarean delivery according to the effects on Apgar scores and acid-base status. Aust NZ Obstet Gynaecol v.39, n.4, p.464-68, 1999.

SHNIDER, S.M.; LEVINSON, G. Anestesia em obstetrícia In: MILlER, R.D. Anestesia. 3.ed. São Paulo : Artes médicas, 1993. p.1829-1874.

SHORT, C.; BUFALARI, A. Propofol anesthesia. Vet Clin Nort
Amer: Small Anim Pratice, v.29, n.3, p.747-777, 1999.

THURMON, J.C. et al. Anaesthesia for special patients: cesarean section patients. In: LUMB \& JONES Veterinary anesthesia. 3.ed. Pennsylvania : Willams \& Wilkins, 1996. Chap.24B, p.818-828.

WATERMAN, A. Accidental hypothermia during anaesthesia in dogs and cats. Veterinary Record, v.96, n.14, p.308-313, 1975.

WILDER-SMITH, O.H.G. et al. Midazolam premedication reduces propofol dose requirements for multiple anesthetic endpoints. Can Journal of Anesthesia. v.48, n.5, p.439445, 2001. 